\title{
A Focus on Powder Diffraction Software
}

The strip chart recorder is dead! (Well, yes, there are still a few powder diffractometers around with them, but better they be in museum exhibits-I worry about the safety of the associated instruments.) Today, nearly everything we do in powder diffraction requires software. The word processor used to type this is generic in nature, but there are wonderful innovations in both commercial and freely-distributed software that change what we can achieve from our experiments.

One goal for the Powder Diffraction Software section in this issue of Powder Diffraction is to highlight software for use with powder diffraction and it features four articles that describe different aspects of the software. As usual, we look to present information that is not found elsewhere. In one case, we have authors taking us behind the scenes in a highly novel commercial code to explain how one aspect of the functionality has been implemented. Yet another describes a new application for that same program. In the others, we get a tour of how new features in new versions of programs will extend the utility of software that may already be in our personal toolbox.
I cannot imagine a future where visualization and analysis software will be less important for powder diffractionists than today, so I would like to use this space to emphasize that this Journal intends to be a venue to educate our community in this important aspect of our craft. Articles may provide an archival mechanism to describe novel algorithms or applications, document the availability of new software or may educate potential users on how to apply software to their problems. Subjects can include commercial software, though in that case care is needed to present a balanced viewpoint as is appropriate for a scholarly publication. I invite you to submit intended publications through the normal editorial process. We are starting to plan another issue with a Powder Diffraction Software section for the summer of 2014; it is not too early to contact me to discuss what you might like to prepare for that issue.

Brian H. Toby Argonne National Laboratory Brian.Toby@anl.gov 